\title{
Highly conductive p-type nanocrystalline silicon films deposited by RF-PECVD using silane and trimethylboron mixtures at high pressure
}

\author{
S.A. Filonovich ${ }^{\mathrm{a}, *}$, H. Águas ${ }^{\mathrm{a}, \mathrm{b}}$, I. Bernacka-Wojcik ${ }^{\mathrm{b}}$, C. Gaspar ${ }^{\mathrm{a}}$, M. Vilarigues ${ }^{\mathrm{c}}$, L.B. Silva ${ }^{\mathrm{a}, \mathrm{b}}$, \\ E. Fortunato ${ }^{a, b}$, R. Martins ${ }^{a, b}$ \\ a Departamento de Ciência dos Materiais, FCT-UNL, Cenimat - I3N, Campus de Caparica, 2829-516 Caparica, Portugal \\ ${ }^{\mathrm{b}}$ Cemop-Uninova, FCT-UNL, Campus de Caparica, 2829-516 Caparica, Portugal \\ ${ }^{\mathrm{c}}$ Dep. de Conservação e Restauro and Centro do Vidro e da Cerâmica para as Artes, FCT-UNL, 2829-516 Caparica, Portugal
}

Keywords:

PECVD

Trimethylboron

nc-Si:H

Doping

Structural properties

\begin{abstract}
A B S T R A C T
In this paper we present a study of boron-doped nc-Si:H films prepared by PECVD at high deposition pressure ( $\geq 4 \mathrm{mbar}$ ), high plasma power and low substrate temperature $\left(\leq 200^{\circ} \mathrm{C}\right)$ using trimethylboron (TMB) as a dopant gas. The influence of deposition parameters on electrical, structural and optical properties is investigated. We determine the deposition conditions that lead to the formation of p-type nanocrystalline silicon thin films with very high crystallinity, high value of dark conductivity $\left(>7(\Omega \mathrm{cm})^{-1}\right)$ and high optical band gap ( $\geq 1.7 \mathrm{eV}$ ). Modeling of ellipsometry spectra reveal that the film growth mechanism should proceed through a sub-surface layer mechanism that leads to silicon crystallization.

The obtained films are very good candidates for application in amorphous and nanocrystalline silicon solar cells as a p-type window layer.
\end{abstract}

(c) 2009 Published by Elsevier Ltd.

\section{Introduction}

P-type hydrogenated nanocrystalline silicon (nc-Si:H) films are attractive for use in amorphous and nanocrystalline silicon optoelectronic devices as a window layer for solar cells or colour light detectors [1]. Those films have high electrical conductivity; high transparency and are more stable under illumination (decreased degradation) than the amorphous counterpart [2,3]. Moreover, in the case of nc-Si solar cells, a highly crystalline window layer facilitates the nucleation and homogeneous growth of the microcrystalline intrinsic layer [4].

Trimethylboron (TMB) has been receiving attention as a better alternative to diborane and methane mixtures for the deposition of p-type silicon carbide films for application in solar cells [5]. TMB is particularly well suited for solar cell deposition process because it has a superior thermal stability than most of the conventional boron sources such as diborane. TMB does not decompose into elementary boron in hot zones without plasma contact, as in the heated part of the gas-inlet and at the gas showerhead, while diborane decomposes in hot zones. Therefore, TMB reduces the risk of boron contamination in hot zones [6]. Moreover, one can obtain films with an optical gap $0.1 \mathrm{eV}$ higher than with diborane [7],

\footnotetext{
* Corresponding author. Fax: +3512 12941365.

E-mail address: filan@fct.unl.pt (S.A. Filonovich).
}

without reducing the electrical conductivity. The other important advantage of TMB confronting with diborane is the fact that TMB is much less hazardous [8].

However, p-type nc-Si:H films are difficult to obtain using TMB. First of all, the presence of boron atoms in a layer reduces the tendency of crystalline growth. Boron has been reported to have a catalytic reaction with the hydrogen resulting in the disruption of the crystal growth due to loss of surface hydrogen coverage [9]. The supplementary carbon atoms (three per TMB molecule) here further decrease the tendency of crystalline growth, favouring amorphous growth. Furthermore, it has been shown that under the same deposition conditions TMB reduces crystalline fraction from $87 \%$ to $53 \%$ as compared to diborane [10].

In this work we investigate the influence of deposition parameters, namely working pressure and plasma power density, on the structural and electronic properties of p-type nc-Si:H thin films using TMB as a dopant gas.

\section{Experimental details}

\subsection{Film deposition}

The films were deposited on $1 \mathrm{~mm}$-thick glass substrates by radio frequency plasma enhanced chemical vapor deposition (RF-PECVD). Before deposition, substrates were heated up to the substrate temperature, $T_{\text {sub }}$, while the base pressure in the chamber 
reached $10^{-6}$ mbar. As gas sources $\mathrm{H}_{2}, \mathrm{SiH}_{4}$ and $\mathrm{TMB}$ were used. The doped samples were prepared at a constant hydrogen dilution, $D_{\mathrm{H}}$, $\left(D_{\mathrm{H}}=F_{\mathrm{H}_{2}} /\left(F_{\mathrm{H}_{2}}+F_{\mathrm{SiH}_{4}}+F_{\mathrm{TMB}}\right) \times 100 \%\right.$, where $F$ s are gas flow rates) of $98 \%$ and $\mathrm{TMB}$-to-silane flow rate ratio $\left(R_{\mathrm{TMB}}=F_{\mathrm{TMB}} / F_{\mathrm{SiH}_{4}} \times 100 \%\right)$ of $0.67 \%$.

In order to optimize the deposition parameters for producing highly conductive films, while preserving high optical gap values, a series of samples were deposited using different substrate temperature, working pressure $\left(p_{\mathrm{w}}\right)$ and $\mathrm{RF}$ power $\left(P_{\mathrm{RF}}\right)$. All the deposition parameters are reported in Table 1.

\subsection{Film characterization}

The films were characterized by spectroscopic ellipsometry (SE) in a wide range (1.5-6.50 eV) with a Horiba Jobin Yvon UVISEL spectroscopic ellipsometer, using a $70^{\circ}$ fixed incidence artgle. Modeling was performed by fitting the data with material references [11] (Fig. 1), using a five-layer model, to determine the crystalline fraction, $X_{\mathrm{CSE}}$, of the films, and its variation with the thickness. This model consisted of dividing the film into five layers: two for the film bulk; one for the sub-surface; one for the surface and one for the surface roughness. In these layers, a variable percentage mixture of two material references, corresponding to the optical properties presented in Fig. 1, plus voids (the software is limited to three materials per layer) was used in order to achieve the lowest error $\left(\chi^{2}\right)$ between measured data point and generated data from the model. Typically, when $\chi^{2}<1$ the model is considered correct. In our case we always had $\chi^{2}<0.5$. The best results were obtained with a variable BEMA mixture of the references for nc-Si, a-Si and voids. With this method we could model the film structure as a function of its thickness, using only three independent variables per sub-layer, making a total of 15 variables to fit the film. Fig. 2 shows a perfect match between the experimental data points, and the plot obtained by fitting. This allowed to elucidate on the film growth mechanism.

Besides that, as an alternative to the use of references, we modeled the film using the Tauc-Lorentz (TL) model [12] in order to estimate the values of the optical gap $E_{\text {opt }}$. We follow this approach to determine $E_{\mathrm{opt}}$ as the data obtained from transmission spectroscopy did not follow the Tauc plot due to the nanocristallinity of the films. The film surface roughness was modelled by fitting the layer with a Bruggeman Effective Medium Approximation (BEMA) of $50 \% \mathrm{TL}$ and $50 \%$ voids.

Raman scattering experiments were performed in the backscattering geometry configuration, using a LabRam 300 Horiba Jobin Yvon with a $632.8 \mathrm{~nm}$ He-Ne laser source at room temperature. The power of the incident beam was small enough $(1.7 \mathrm{~mW})$ to avoid re-crystallization of the films. The crystalline volume fraction $X_{\text {CRS }}$ was determined as: $X_{\mathrm{CRS}}=\left(I_{\mathrm{C}}+I_{\mathrm{I}}\right) /\left(I_{\mathrm{C}}+I_{\mathrm{I}}+I_{\mathrm{A}}\right) \times 100 \%$, where $I_{C}$ and $I_{I}$ are the integrated intensities of the $520 \mathrm{~cm}^{-1}$ and the $510 \mathrm{~cm}^{-1}$ contribution of the transverse optical (TO) peak of the Raman spectrum, whereas $I_{\mathrm{A}}$ refers to the amorphous contribution at $480 \mathrm{~cm}^{-1}$ [13].

For the dark conductivity $\sigma_{\mathrm{d}}$ measurements, $4 \mathrm{~mm}$ long and $1 \mathrm{~mm}$ apart coplanar aluminium contacts were deposited using an e-beam metallization system. The activation energy was calculated

Table 1

Deposition conditions.

\begin{tabular}{ll}
\hline$D_{\mathrm{H}}(\%)$ & $97-98$ \\
$R_{\mathrm{TMB}}(\%)$ & $0 ; 0.67$ \\
$T_{\mathrm{sub}}\left({ }^{\circ} \mathrm{C}\right)$ & $100-200$ \\
$p_{\mathrm{w}}(\mathrm{mbar})$ & $2.7-6.0$ \\
$P_{\mathrm{RF}}(\mathrm{W})$ & $120-180$ \\
\hline
\end{tabular}

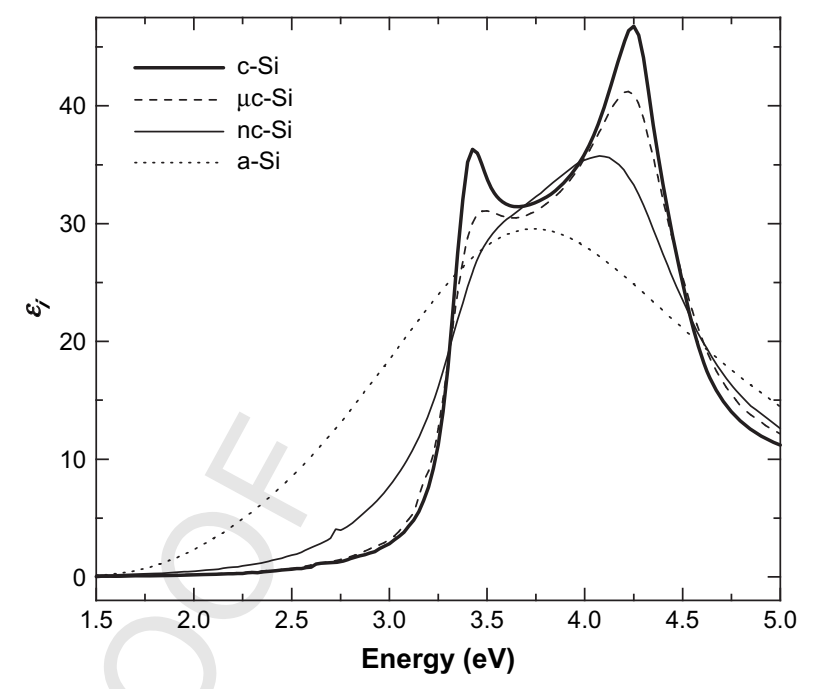

Fig. 1. Spectroscopic Ellipsometry reference data of the imaginary part of the dielectric function $\left(\varepsilon_{\mathrm{i}}\right)$ for monocrystalline (c-Si), microcrystalline ( $\mu \mathrm{c}-\mathrm{Si}$ ), nanocrystalline (nc-Si) and amorphous (a-Si) silicon.

from the temperature dependence of $\sigma_{\mathrm{d}}$ in the range $363-253 \mathrm{~K}$ using $\sigma_{\mathrm{d}}=\sigma_{0} \exp \left[-E_{\mathrm{a}} / k_{\mathrm{B}} T\right]$, where $k_{\mathrm{B}}$ is the Boltzmann constant. The mobility and the charge carrier concentration of selected samples were determined by Hall measurements, using a commercial set-up (Biorad 500).

\section{Results and discussion}

Deposition of nc-Si:H films by PECVD with high crystalline volume fraction requires typically very high values of hydrogen dilution. As well, a high deposition pressure (4.0-6.7 mbar) facilitates growth of nc-Si:H. Prior to deposition of doped nc-Si:H films, the deposition conditions for intrinsic nc-Si:H films were optimized for the substrate temperature of $200{ }^{\circ} \mathrm{C}$. Hydrogen dilution of $97 \%$ was high enough to produce nc-Si films with high crystalline volume fraction $\left(X_{\mathrm{CRS}}=65 \%\right)$. However, when a small amount of TMB was added to the gas mixture, while keeping $D_{\mathrm{H}}$ value at $97 \%$, the obtained Si film exhibited structural and electroniç्र properties typical of doped amorphous silicon films. Therefore, an increase of

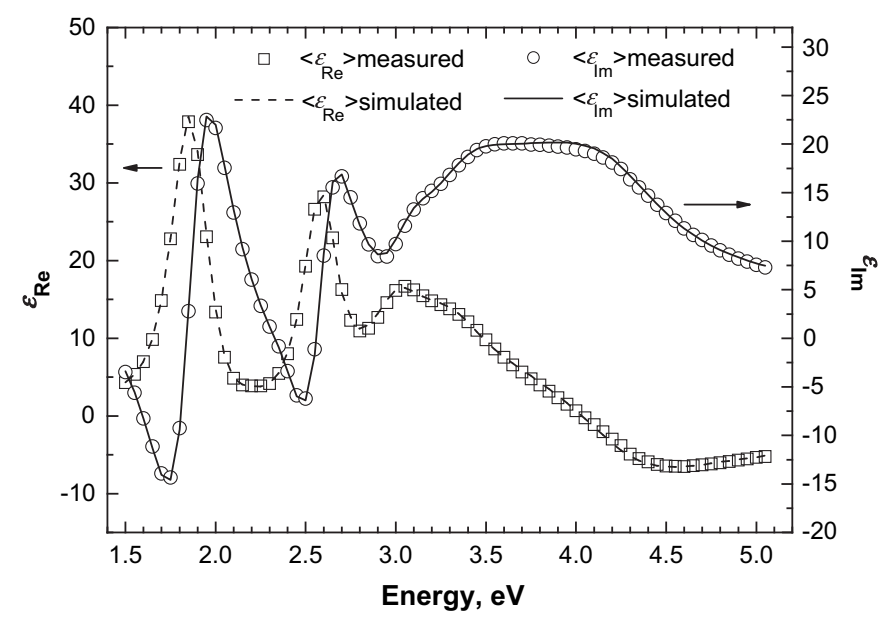

Fig. 2. Experimentally measured points and corresponding fitted curve from the dielectric function model. 
hydrogen dilution (up to 98\%) was required to achieve the growth of nc-Si in the presence of TMB.

An optimal value of TMB-to-silane flow rate ratio for obtaining a thin film with the highest doping efficiency was determined to be $0.67 \%$ (subject of another investigation). The dark conductivity, $\sigma_{\mathrm{d}}$, of the resulting film was $0.6(\Omega \mathrm{cm})^{-1}$, with activation energy, $E_{\mathrm{a}}$, $41 \mathrm{meV}$. Crystalline volume fraction deduced from Raman spectroscopy (RS) and SE was $72 \%$ and $85 \%$, respectively. It was previously reported [14] that the crystalline fraction calculated from RS by the method mentioned in Introduction $\operatorname{can}_{\lambda}$ give a lower crystalline fraction than real. On the other hand, in SE the use of references for modeling corresponding to materials that could differ in grain size from our actual films can also lead to an overestimation of the crystalline fraction. However, we believe that the SE analysis is readily accurate since the $\chi^{2}$ obtained with simulations was always inferior to 0.5 . Despite a very high value of $X_{C}$, the optical band gap of the film deduced from the TL model of the film was estimated to be $1.77 \mathrm{eV}$. One should expect lower optical band gap of nc-Si:H films with very small amorphous volume fraction (tending to $1.1 \mathrm{eV}$ as for monocrystalline silicon). However, in our case, the presence of carbon in the nc-Si:H film widens the optical gap.

The growth mechanism of intrinsic and doped nc-Si films was highly dependent on the substrate temperature, hydrogen dilution and power density used [15]. While for intrinsic films, the best crystallinity was achieved at high deposition temperature $\left(>250^{\circ} \mathrm{C}\right)$, for the doped nc-Si films, low deposition temperature might be beneficial $[16,17]$. Several depositions were performed at substrate temperatures in the range $100-200{ }^{\circ} \mathrm{C}$, maintaining other deposition conditions constant $\left(P_{\mathrm{RF}}=180 \mathrm{~W}, p_{\mathrm{w}}=5.33 \mathrm{mbar}\right)$, and it was found that for the samples deposited at $150^{\circ} \mathrm{C}, \sigma_{\mathrm{d}}$ was the highest $\left(7.1(\Omega \mathrm{cm})^{-1}\right)$. This is due to the fact that at this deposition temperature obtained nc-Si film possesses the highest crystalline volume fraction (as defined by RS and SE). However, the optical band gap of this film is still maintained at a high level, $E_{\text {opt }}=1.72 \mathrm{eV}$. As well, this film shows very high Hall mobility and carrier concentration at room temperature: $0.56\left(\mathrm{~cm}^{2} / \mathrm{Vs}\right)$ and $5.7 \times 10^{19} \mathrm{~cm}^{-3}$, respectively.

For further optimization of the doping efficiency a series of depositions was performed with varied values of the gas pressure and power of plasma, while maintaining the substrate temperature at $150{ }^{\circ} \mathrm{C}$. Fig. 3 shows the distribution of $\sigma_{\mathrm{d}}$ values in the coordinate grid of RF power and gas pressure. It was found that highly conductive films were obtained in high power and high-pressure regime. However, the films grown in these conditions exhibit high

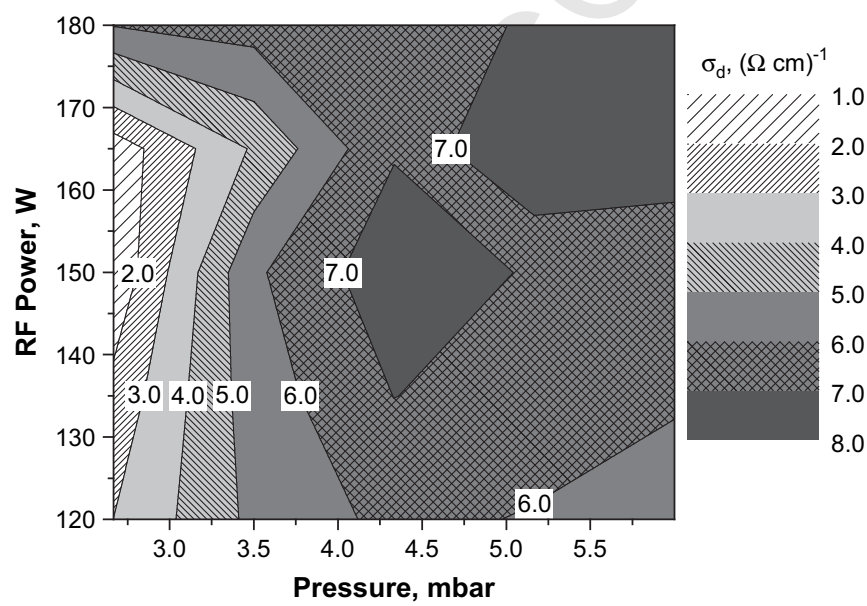

Fig. 3. Variation of dark conductivity as a function of RF plasma power and deposition pressure. inhomogeneities as far as thickness and structure are concerned. Thus, those films are not suitable for application as a window layer in solar cells. The optimal deposition conditions for growing homogeneous films (5\% of thickness distribution for the area $\left.10 \times 10 \mathrm{~cm}^{2}\right)$ with the best electrical $\left(\sigma_{\mathrm{d}}=7.4(\Omega \mathrm{cm})^{-1}\right.$, $\left.E_{\mathrm{a}}=18 \mathrm{meV}\right)$ and optical $\left(E_{\mathrm{opt}}=1.77 \mathrm{eV}\right)$ properties correspond to the use of a RF power of $150 \mathrm{~W}$ (power density: $187 \mathrm{~mW} / \mathrm{cm}^{2}$ ), working gas pressure of $4 \mathrm{mbar}$ and $T_{\text {sub }}=150{ }^{\circ} \mathrm{C}$.

The SE simulation analysis allowed also a better understanding of the hydrogen dilution role, to the growth mechanism of the nanocrystalline films. From SE modeling we could observe that there is no incubation layer in contact with the substrate, but in fact, the films are more crystalline nearer to the substrate than to the surface (Fig. 4 ). This variation of morphology was determined by modeling the film with five layers. The results are shown in Fig. 4 for a film with the thickness of $140 \mathrm{~nm}$. The two bulk layers are almost identical and present a high crystalline fraction, showing only a very small variation between them. The top layer corresponds to surface roughness, but we choose not to represent it in Fig. 4 to allow a clearer view of the trends. The surface layer consists of deposited material with a higher amorphous fraction than the bulk, but already with the incubation of some nanocrystalline grains that could have grown from incorporation of small nanoparticles from the plasma [18]. Bellow this layer is a sub-surface layer, where the void fraction reaches a maximum of more than $80 \%$ for a very small thickness range of about $5-10 \mathrm{~nm}$. We believe that this layer is responsible for the re-crystallization of the growing film: as the layer moves to the surface leaving behind a recrystallized film as it grows. We believe that this mechanism takes place by in-diffusion of hydrogen in to the growing film, causing the re-crystallization of film in a highly porous and hydrogen-rich a$\mathrm{Si}: \mathrm{H}$ sub-surface layer. In-situ studies of the exposure of a-Si:H to a hydrogen plasma have shown that hydrogen can diffuse very rapidly into an a-Si:H network and modify the structure of the film over a thickness of a few tens of nanometers [19,20].

These results indicate that the growth of these boron-doped nanocrystalline films occurs through a sub-surface reaction mechanism, as also observed for the intrinsic silicon films grown at high pressure and high hydrogen dilution conditions [21,22].

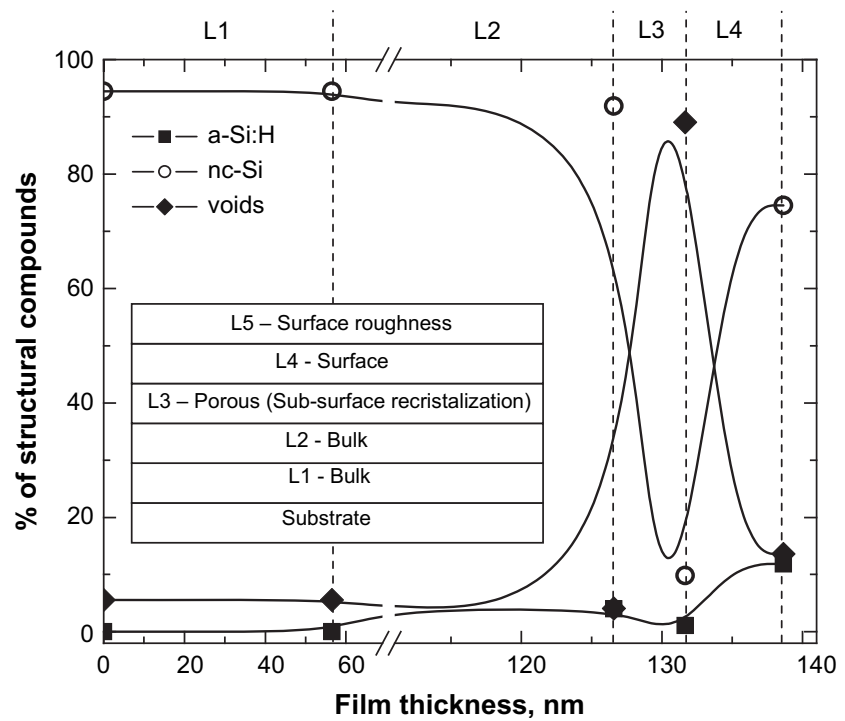

Fig. 4. Representation of structural variation with the film thickness, extracted from fitting the SE data with a five-layer model (please, note the difference in the scale before and after the brake). The insert represents a sketch of the model. The surface layer corresponding to roughness is not represented, for a clear data view. 
A comparative analysis (TEM, SEM) is underway and is expected to confirm this conclusion. Moreover we deposited very thin films with $20 \mathrm{~nm}$ and found them to present some nanocrystallinity attested by SE measurements.

\section{Conclusion}

The influence of deposition conditions (namely working gas pressure, RF power and substrate temperature) on the structural and electrical properties of boron ${ }_{\Sigma}$ doped nanocrystalline silicon was systematically studied. We have shown that highly conductive p-type nc-Si:H $\left(\sigma_{\mathrm{d}}=7.4(\Omega \mathrm{cm})^{-1}\right)$ can be obtained at $150{ }^{\circ} \mathrm{C}$ by mixing TMB, silane and hydrogen. These films have a very high value of crystalline fraction, however the optical band gap is still high $(1.77 \mathrm{eV})$ due to presence of carbon in the film. Such properties of the p-type Si film make this material a very good candidate to be used as a window layer in solar cell applications and colour light detectors.

Based on the spectroscopic ellipsometry fitting data, we proposed a growth model for the p-type nc-Si:H film, which includes a sub-surface incubation layer, instead of the typical incubation layer at the-interface substrate - film

\section{Acknowledgements}

The authors acknowledge the support from Fundação para a Ciência e Tecnologia through the through pluriannual contract with CENIMAT and projects PTDC/EEA-ELC/74236/2006, and PTDC/ FIS/74274/2006.

\section{References}

[1] Hu ZH, Liao XB, Diao HW, Cai Y, Zhang SB, Fortunato E, et al. Journal of NonCrystalline Solids 2006;352:1900-3;
See also Martins R, Baptista P, Raniero L, Doria G, Silva L, Franco R, et al. Applied Physics Letters 2007;90:023903-5.

[2] Rath JK, Schropp REI. Solar Energy Materials and Solar Cells 1998;53: 189-203.

[3] Filonovich SA, Alpuim P, Rebouta L, Bouree, Soro YM. Journal of NonCrystalline Solids 2008;354:2376-80.

[4] Matsui T, Kondo M, Matsuda A. Journal of Non-Crystalline Solids 2004;338340:646-50.

[5] Lee HJ, Sazonov A, Nathan A. Materials Research Society Symposium Q2 Proceedings 2007;989. 0989-0A21-07,

[6] Cabarrocas PRoca i, Kumar S, Drévillon B. Journal of Applied Physics 1989;66:3286.

[7] Lloret A, Wu ZY, Theye ML, El Zawawi I, Siefert JM, Equer B. Applied Physics A 1992;55:573-81.

[8] Blanchard WR, Gernhardt RC, Kugel HW, LaMarche PH. 19th IEEE/NPSS Symposium on Fusion Engineering, Proceedings 2002:226-9.

[9] Perrin J, Takeda Y, Hirano N, Takeuchi Y, Matsuda A. Surface Science 1989;210:114.

[10] Eser E, Hegedus SS, Buchanan WA. AIP Conference Proceedings 1999;462: 254-9.

[11] Reference spectra are supplied by Jobin Yvon software. Origin of the references:. In: Palik ED, editor. Handbook of optical constants of solids. Elsevier; 1998;

Aspnes DE, Studna AA. Physical Review B 1983;27:985

[12] Jellison GE, Modine MF. Applied Physics Letters 1996;69:371.

[13] Kaneko T, Wakagi M, Onisawa K, Minemura T. Applied Physics Letters 1994;64:1865.

[14] Ledinský M, Fekete L, Stuchlík J, Mates T, Fejfar A, Kočka J. Journal of NonCrystalline Solids 2006;352:1209.

[15] Zhang S, Liao X, Xu Y, Martins R, Fortunato E, Kong G. Journal of Non-Crystalline Solids 2004;338:188-91.

[16] Kondo M, Nasuno Y, Mase H, Wada T, Matsuda A. Journal of Non-Crystalline Solids 2002;299-302:108-12.

[17] Alpuim P, Chu V, Conde JP. Journal of Vacuum Science \& Technology A 2003;21:1048

[18] Cabarrocas PRoca i, Morral AFontcuberta i, Lebib S, Poissant Y. Pure and Applied Chemistry 2002;74:359.

[19] Otobe M, Oda S. Japanese Journal of Applied Physics 1992;31:L-1388.

[20] Yamasaki S, Das UK, Yasuda T. Journal of Non-Crystalline Solids 2002;185:299-302.

[21] Cabarrocas PRoca i, Morral AFontcuberta i, Kalache B, Kasouit S. Solid State Phenomena 2003;93:257-68.

[22] Zhang S, Liao X, Raniero L, Fortunato E, Xu Y, Kong G, et al. Solar Energy Materials and Solar Cells 2006;90:3001-8. 